Wuppertal Institut für Klima, Umwelt, Energie $\mathrm{GmbH}$

Hans-Jochen Luhmann

\title{
Der (Flug-)Verkehr nimmt zu - auch in Zeiten des Klimawandels: Wie kommt es zu diesem Paradox?
}

Ursprünglich veröffentlicht als:

Hans-Jochen Luhmann (2010):

Der (Flug-)Verkehr nimmt zu - auch in Zeiten des Klimawandels: Wie kommt es zu diesem Paradox?

In: Leviathan, Jg. 38, Nr. 4, S. 475-488 
Hans-Jochen Luhmann

\section{Der (Flug-)Verkehr nimmt zu - auch in Zeiten des Klimawandels: Wie kommt es zu diesem Paradox?}

\section{Kurzfassung}

Zum Flugverkehr als klimapolitischen ,Ausreißer', wird zweierlei gefragt: (1) Nach dem zentralen Grund für die nach oben gerichtete Sonderstellung des (Flug-)Verkehrs. (2) Wie im konkreten Falle, bei der Erweiterung der Kapazität des Flughafen München, die klimapolitischen Randbedingungen marginalisiert werden.

Schlagworte:

Flugverkehr; Verkehrsinfrastruktur; Raumordnungsrecht; Treibhausgasemissionen; Emissionshandel

\section{Abstract}

Aviation is the fastest growing sector, measured in GHG emissions. Subject are two issues: (1) What is the key reason for the extraordinary growth inclination of transport in general and of aviation especially? (2) How is it managed, that the process of arguing for an capacity expansion at Munich airport is biased, that any limitation due to the climate change challenge is systematicly neglected or marginalised?

Aviation; Transport-Infrastructure; planning law; GHG emissions; EU Emissions Trading System

Autorenangaben:

Dr. Hans-Jochen Luhmann

Wuppertal Institut für Klima, Umwelt, Energie GmbH

Döppersberg 19; D-42103 Wuppertal

jochen.luhmann@wupperinst.org

\section{Die Dynamik des (Flug-)Verkehrs und Ohnmacht der Klimapolitik}

Der kategorische Imperativ in Zeiten des Klimawandels lautet: Wir müssen den anthropogenen Klimawandel zum Stillstand bringen. Das wurde im Juni 1992 mit Art. 2 Klimarahmenkonvention (UNFCCC) im globalen Konsens beschlossen. Dazu ist es notwendig, die Emissionen von Treibhausgasen bis 2050 auf etwa 20\% des heutigen Niveaus zurückzuführen - das ist in Kopenhagen im Dezember 2009 als globaler Konsens formuliert worden. Die Rückführung der Emissionen ist eine Aufgabe aller Sektoren von Wirtschaft und Gesellschaft, was konzeptionell zumindest auch bei den meisten Sektoren gelingt. Der Verkehr allerdings erweist sich als ,Ausreißer', nur bei ihm geht es schon optional nur nach oben. Welches sind die Gründe für die Sonderrolle des Verkehr?

Dafür finden sich im wesentlichen zwei Antworten aus den zuständigen Feldern der Wissenschaft. Beide Antworten basieren auf der Unterscheidung von ,Verkehr' und ,Mobilität'. Die soziologische Verkehrsforschung weist unter dem Titel Zusammenhang von Mobilität und Moderne auf das Phänomen hin, dass in modernen Gesellschaften anders als in vormodernen Gesellschaften nicht nur die Eliten ,mobil' sind. Modernisierung bedeutet demnach ,Beschleunigung' und zugleich die Verbreiterung des Spektrums der Subjekte, die Verkehrs- 
leistungen in Anspruch nehmen. Es gilt als ,Demokratisierung' von Mobilität (vgl. Canzler, Knie (1998); Freudendahl-Pedersen (2005), Hartwig, Mautz, Rosenbaum (2001), Kuhm (1997), Rammler (2001) und Urry (2007)). Damit identifiziert die Verkehrssoziologie den Kern der Hartnäckigkeit, mit der sich der Personen-Verkehr jeglichen Verzichts- und Reduktionsappellen widersetzt. Unterstützt wird diese Sichtweise durch die ökonomische Theorie der Produktivität, die unterstellt, dass die Globalisierung die Effizienz der Produktion (auch) physischer Güter durch Ausdehnung der räumlichen Reichweite der Arbeitsteilung erhöht und eine erhöhte Nachfrage nach Güterverkehrsleistungen deshalb sowohl zu erwarten als auch gerechtfertigt sei.

Auf dem zweiten Feld, den technischen Verkehrswissenschaften, hält man die Nachfrage nach Verkehrsleistungen nicht für ein hinzunehmendes historisches Phänomen sondern für ,produziert'. ,Mobilität', Schlüsselbegriff der soziologischen Theorie, erweist sich dann als abgeleitetes Phänomen, ist kein eigentliches Bedürfnis mehr. Der damit freigewordene Begriff ,Mobilität' wird in dieser Szene der Verkehrswissenschaft dann neu besetzt: Mobilität wird hier als Bedarfskategorie besetzt, zu verstehen als Bedürfnis nach ,Erreichbarkeit'. In Verron et al. (2005) z.B. wird die Verkehrsentstehung konzeptionell den Teilursachen Siedlungsentwicklung, Handelsverflechtung, Lebensstil und letztlich eben auch der Verkehrsinfrastruktur zugerechnet.

Wenn Verkehr selbst als Bedürfnis verstanden wird, dann haben wir es mit einer Kultur permanenter Fluchtreflexe zu tun, und es stellt sich die Frage: Vor was fliehen die Menschen in großen Scharen, wenn sie zu Millionen in die Urlaubsgebiete ziehen? Von diesem Rätsel bis zu aussichtsreichen Handlungskonzepten zu kommen, ist ein weiter Weg. Die zweite Definition hingegen erweist sich offensichtlich insofern als vorteilhaft, weil sie Handlungsspielräume für Steuerungsintentionen aufzeigt.

Innerhalb dieses als Ganzen problematischen Verkehrssektor gibt es noch eine Steigerung. Am problematischsten sind diejenigen Verkehrsmittel, die ihren Treibstoff mit sich führen, , an Bord' haben: eben ,Auto'-Mobile im eigentlichen Sinne. Dazu zählen auch die Flugzeuge. Kein Bereich innerhalb des Verkehrssektors weist so hohe projizierte und in der Folge auch verfolgte Wachstumsraten seiner Treibhausgasemissionen auf wie der Flugverkehr. Das Postulat, dass es für ihn ein Konzept geben müsse, wie er sich einer Welt angepasst haben wird, die das Klimaproblem gelöst hat, ist deswegen von vergleichbar hoher Bedeutung, ist gleichsam das sektorale Pendant zum Postulat der Einbindung des Staates USA in ein globales Klimaregime der Staaten. Beide sind Schlüsselfaktoren für die Klimapolitik, die USA und die Luftverkehrsbranche. Und in beiden ist der Widerstand sehr stark: Sie vermögen nämlich ihre Rolle in eine Welt mit einem Klimagasausstoß nahe Null bislang nicht zu erkennen. Um Konsistenz bemüht leugnen ihre Vertreter lieber die Herausforderung oder spielen auf Zeit: Später werde alles gut werden - vgl. zur Veranschaulichung den Kasten. 
Das globalpolitisch mandatierte Ziel ,minus 50\% bis 2050' will die Luftfahrtbranche nach Ausweis der Airbus-Präsentation, präsentiert beim International Transport Forum in Leipzig 2010, angeblich in der gezeigten Weise erreichen: Ab 2040 gehe es plötzlich abwärts, ein Bezug zur Lebensdauer des Fluggeräts wird nicht aufgezeigt. Plötzlich, um das Jahr 2040 herum beginnend, werde die Branche ihre, eigentlich' noch flugtauglichen Geräte in nur 10 Jahren gegen radikal ,moderne‘ auswechseln. Das will diese Präsentationsgrafik dem politischen Publikum weismachen. Die Botschaft in den politischen Raum lautet: Noli me tangere! Lass mich noch dreißig Jahre Schmuddelkind bleiben! Ich werde später von alleine brav.

\section{Aviation: Emissions reduction roadmap}
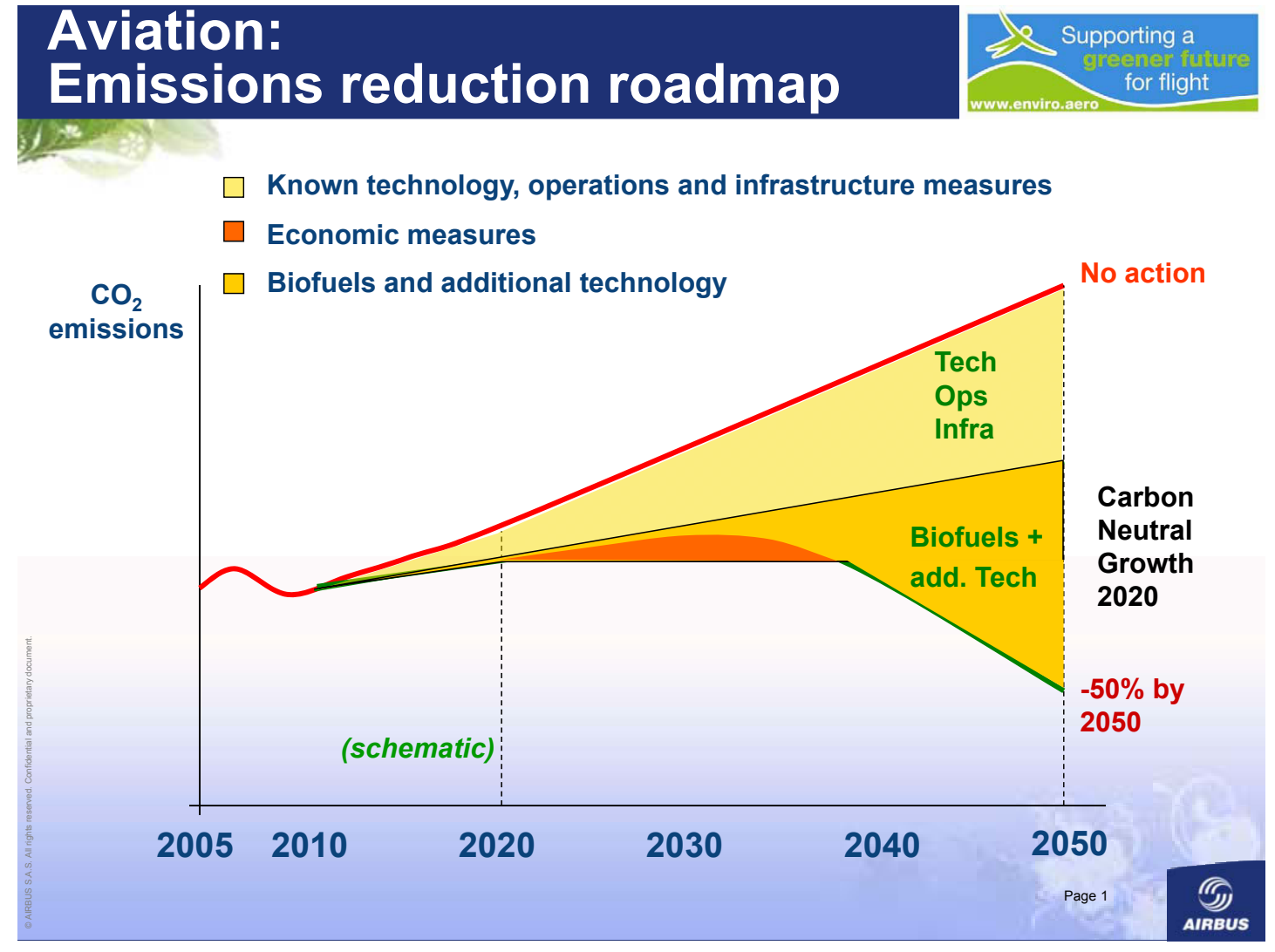

Quelle: http://www.internationaltransportforum.org/2010/pdf/Dumas.pdf

Der Flugverkehr hat mit dem Straßenverkehr eines gemeinsam: Er erscheint wie eine Flut, welche immer höher steigt und den gesetzten Deich an einigen Stellen aufweicht, wo dann das Wasser durchsickert. Repariert man den Deich an einer Stelle, so sucht sich das Wasser andernorts einen neuen Durchgang. Verstärkt man den Deich hingegen insgesamt und baut ihn höher, so hilft auch das nicht auf Dauer: Die Flut wächst und überwindet nach einiger Zeit auch die Höhe des neu geschaffenen Scheitels. In einer solchen Situation hilft offenkundig nur eines: den Anstieg des Wassers zu stoppen. Das nötigt dazu zu fragen, aus welchem Grunde das Wasser steigt und wie der Stopp des Anstiegs erreicht werden kann.

Vor einer Antwort auf eine solche Frage steht Kritik: Es sind nicht die Sachen selbst, der Verkehr und die Nachfrage nach ihm, die zu dieser laokoonischen Situation geführt haben. Es ist vielmehr das Konzept unseres kollektiven Umgangs mit diesen Sachen, welches uns in die Irre geführt hat. Selbstbezug in der Kausalität ist die Struktur des Problems, Selbstkritik ist damit angesagt; und da nichts schwerer fällt als diese, hat man sich die spezifischen Schwierigkeiten, die bei einer solchen Diagnose zu erwarten sind, vorab ins Bewusstsein zu rufen. 
Die Diagnose in Kap. II und III könnte kränkend wirken, aber sie könnte dessen ungeachtet wahr sein.

\section{Verkehrsinfrastrukturpolitik: Das ,Prognose und Bereitstell'-Prinzip ${ }^{1}$}

Das Konzept von Prognose und Bereitstellung basiert auf der begrifflichen Trennung von Verkehr und Rahmenbedingungen des Verkehrs, ganz nach dem scholastischen Schema von Substanz und Akzidenzien. Flugverkehr wie motorisierter Straßenverkehr sind aber mit ihren je spezifischen Umweltbeeinträchtigungen untrennbar verbunden. Wachsender Verkehr ist deswegen mit einer wachsenden Umweltbeeinträchtigung verbunden. ${ }^{2}$ Dies ist eine Einsicht, die durch die vorgenommene Trennung ausgehebelt wird. Diese Einsicht impliziert aber, dass es nicht genügt, einen umweltrechtlichen Rahmen für Akzidenzien eines ungehindert anschwellenden Verkehrs zu setzen. Das nämlich ist ein illusorischer Akt, vergleichbar dem Deichbau. Versucht man es dennoch, dann entspricht das Ergebnis der jüngst vorliegenden Erfahrung ansteigender Fluten: Der gesetzte Rahmen ist dann, wie ein Deich, eines Tages nicht mehr zu halten. Soll er gehalten werden, müsste er wachsen. Ein wachsender Rahmen aber ist kein containment, sondern ein Paradox: Der Rahmen verliert seine eigentliche Funktion.

Will man dem Paradox entkommen, so ist es erforderlich, eine konzeptionelle Konsequenz zu ziehen: Das Anschwellen des Verkehrs selbst muss als Problem angesehen werden. Gesundheits- und Umweltschutz, insbesondere aber der Klimaschutz, nötigen zu einem Wechsel: weg von einer Betrachtung von Relationen, weg von spezifischen Werten, hin zu einer Betrachtung absoluter Mengen. Dieser Wechsel in der Betrachtung dient einem Konzept, das nach Möglichkeiten der Beendigung des Zuwachses des Verkehrs zu suchen erlaubt. Verkehrspolitik und Verkehrsinfrastrukturplanung werden bis heute von einem Konzept beherrscht, welches selten explizit formuliert wird. Dem Rat von Sachverständigen für Umweltfragen (SRU) verdanken wir eine überzeugende Reformulierung. Demnach verstehen Fachliteratur und Rechtsprechung das deutsche Recht des Errichtens von Verkehrsinfrastrukturen so, dass es auf jener Unterscheidung zwischen dem Verkehr selbst und seinen externen Effekten aufbaue. Auf diese Weise ist es möglich, ,den an sich, guten' ... Verkehr von seinen ,unerwünschten' Nebenfolgen zu trennen, und Strategien zu konzipieren, deren Umsetzung die Bewältigung eines steigenden Verkehrsaufkommens mit Zielen einer Reduzierung der Nebenwirkungen in Einklang zu bringen versucht.“ (SRU 2005, Ziff 134) Dieser diagnostische Satz des SRU spiegelt in meisterhafter Präzision die Ambivalenz der beteiligten Fachleute: Diesem Satz vermögen alle Experten zuzustimmen. In zwei Lager aufteilen würden die Fachleute sich erst dann, wenn an den Ausdruck ,Ziele einer Reduzierung der Nebenwirkungen“ die Gretchenfrage gestellt wird: Folgst Du einem relativen oder einem absoluten Verständnis dieser Formel? Geht es Dir um spezifische oder um absolute Reduktionen? Das Konzept erlaubt, eine ständig wachsende Flut von Verkehr und damit von Umweltbeeinträchtigungen

Gerd Winter (2010). Ausgeführt hat er sie am Beispiel des Straßenverkehrs.

2 Dem widerspricht nicht, dass es in einzelnen Parametern der Umwelt- und Gesundheitsbelastungen auch zu einem absoluten Rückgang bei wachsendem Verkehr gekommen ist und also kommen kann. Die Aussage ist lediglich, dass dies nicht generell möglich ist, sondern nur unter bestimmten Bedingungen und in zeitlich begrenzten Situationen. Der logische Status der Aussage ,Wachsender Verkehr ist mit einer wachsenden Umweltbeeinträchtigung verbunden“ ist nicht der einer All-Aussage sondern der einer generellen Aussage. Dass die PR diese Differenz übergeht, gehört zu der Kunstfertigkeit, derentwegen sie bezahlt wird. 
zuzulassen, und es erlaubt auch, dass diese Gretchenfrage umgangen wird, dass sie nicht, zumindest nicht handlungsrelevant, gestellt wird.

Das leistet das Prinzip Prognose und Bereitstellung (,predict and provide'), das für den ,guten' Verkehr gilt. Erst wird im Rahmen der Infrastrukturplanung die Nachfrage nach Verkehrsleistungen prognostiziert, und wenn sich zeigt, dass die vorhandene Flugverkehrsinfrastruktur nicht hinreichend dimensioniert ist, den Zuwachs zu befriedigen, dürfen vorhandene Kapazitäten erweitert werden. Im rechtsdogmatischen Konzept der Planfeststellung wird dieses Prinzip auf der Stufe der Planrechtfertigung wirksam: Ein Vorhaben ist gerechtfertigt, wenn eine ungedeckte Verkehrsnachfrage ,besteht'. Über die Feststellung des ,Bestehens' einer Lücke wird gemäß dem Kriterium in der ständigen Formel des Bundesverwaltungsgerichts (BVerwG) entschieden: Das Erweiterungsvorhaben muss ,vernünftigerweise geboten' sein.

Basiert ein Ausbauvorhaben auf einer, vernünftigen' Prognose, dann ist es faktisch nicht mehr aufzuhalten. Hat ein Vorhaben einmal den Filter der Planrechtfertigung durchlaufen, wird es auf der zweiten Stufe, der Abwägung, anderen Belangen nicht mehr gegenübergestellt. Das Vorhaben ist nun als ,an sich gut“" anerkannt und wird nur noch im Hinblick auf seine Nebenfolgen geprüft. Damit wird, um im Bild zu bleiben, der Anstieg der Verkehrsflut zugelassen, ohne Rücksicht auf die Kapazität des vorhandenen Deichsystems.

\section{Das Konzept, welches die (Verkehrs-)Flut steigen lässt}

Rein rechtsdogmatisch gesehen kann das Vorhaben zwar noch scheitern, praktisch aber kommt es in aller Regel nicht dazu. Zu einer Abwägung dessen, ob das Ausbauvorhaben den verbleibenden zusätzlichen Gesamtschaden für die Umwelt rechtfertigt, ob der zuzulassende Pegelanstieg für das bestehende Deichsystem verkraftbar ist oder ob ein Ausbau der Deiche dann die Konsequenz zu sein hat, kommt es meistens nicht mehr, weil der Nutzen zusätzlichen Verkehrs bereits als auf der Planrechtfertigungsstufe abgeprüft gilt und deshalb auf der Abwägungsstufe nicht mehr in Frage gestellt wird. Das entbehrt der Logik, denn ein Abgleich des relativen Gewichts von Belangen ist nur möglich, wenn beide Seiten ins Verhältnis gesetzt werden. Das BVerwG aber hat sich dieser Einsicht (der Vernunft) bislang verschlossen: Wie dringlich und gewichtig der zusätzliche Verkehrsbedarf angesichts entgegenstehender Belange ist, wird nicht überprüft. Hier haben wir einen entscheidenden Punkt: Es ist das planungsrechtliche Konzept, welches zwangsläufig zum (Verkehrs-) Wachstum führt.

In Bildsprache formuliert: Wer Straßen sät, wird Verkehr ernten - und wer Startbahnen baut, wird auch zusätzlichen Flugverkehr bekommen. Formuliert für die Verwaltungsebene bedeutet das: Wer einen von Interessenvertretern projizierten Zuwachs an Verkehrsnachfrage nicht zur Abwägung mit den Folgen, etwa den dadurch ausgelösten Belastungen für die Umwelt, zulässt und somit den Bau von Verkehrsinfrastrukturen gegenüber anderen Belangen privilegiert, erntet wachsende Verkehrsinfrastrukturen, vulgo ,Straßen' oder Startbahnen. Der Kreis schließt sich: Die Tendenz des sich wie Unkraut verbreitenden ,Säens von Straßen' ist Konsequenz eines höchstrichterlich etablierten Verfahrensgrundsatzes in der Genehmigung von prognostisch begründeten Anträgen auf Ausbau von Verkehrsinfrastrukturen.

Die Flut des Anstiegs des Verkehrs erklärt sich aus dieser aktuellen Lage der Rechtskultur, und aus ihr erklärt sich zugleich die hohe Bedeutung der Qualitätssicherung einer Verkehrsnachfrageprognose. Das rechtlich etablierte Qualitäts-Kriterium stellt auf, Vernunft' ab. Die Wissenschaft gilt definitorisch als Hüter der, Vernunft'. Folglich ist die Prüfung der Qualität 
einer kommerziell zustande gekommenen Verkehrsprognose, die ein Ausbauvorhaben rechtfertigen soll, seitens einer wissenschaftlichen Einrichtung naheliegend. Diese selbst wird in aller Regel von spezialisierten Verkehrs-Consulting-Unternehmen im Auftrag des an einem Ausbau interessierten Unternehmens erarbeitet.

\section{Das Gutachten zur Begründung der Notwendigkeit einer dritten Startbahn am Flugha- fen München und seine Qualitätssicherung - völlige Ausblendung des Klimaproblems}

Die Tendenz eines Verkehrswachstums ist somit im Konzept der Verkehrsinfrastrukturplanung und -durchsetzung verankert. Was für den allgemeinen Fall festgestellt wurde, lässt sich beispielhaft nachverfolgen und anschaulich machen am Vorgang der Vorbereitung der Genehmigung einer dritten Startbahn des Flughafens München (MUC). Im Fokus der folgenden Darstellung steht die Berücksichtigung der Klimaherausforderung in diesem ersten Verfahren, das durchgeführt wurde, nachdem die Staatsspitze Deutschlands sich für ein Emissionsminderungsziel bis $2050 \mathrm{um}$,mindestens“ (!) 80 Prozent ausgesprochen hat.

Das Ausgangsdokument, die Prognose zur Begründung eines Nachfragezuwachses, welcher eine Kapazitätserweiterung für den Flughafen München notwendig erscheinen lässt, wurde von Intraplan Consult GmbH erarbeitet und im Juli 2007 der Genehmigungsbehörde vorgelegt. Diese Behörde, die Regierung von Oberbayern (bzw. das Luftamt Südbayern), hat anschließend vom Institut für Verkehrsplanung und Logistik der Technischen Universität Hamburg-Harburg (TUHH), also einer wissenschaftlichen Einrichtung, in einer eigenen Studie die Qualitätssicherung der Prognose durchführen lassen.

$\mathrm{Zu}$ den Ergebnissen der TUHH-Untersuchung zählte die Feststellung eines Nachbesserungsbedarfs an einer bestimmten Stelle der Intraplan-Prognose. Im Wortlaut heißt es:

„Vor diesem Hintergrund empfehlen wir die Integration von Szenarien, anhand derer differenzierte Entwicklungspfade von Wirtschafts- und Ölpreisentwicklung aufgezeigt werden können. Für einen solchen Schritt ist es sinnvoll, durch ein spezialisiertes Wirtschaftswissenschaftliches Forschungsinstitut (z.B. das Hamburgische WeltWirtschaftsInstitut) ... eine ... Einschätzung der möglichen weiteren wirtschaftlichen Entwicklung bis zum Jahr 2020 (mit einem Ausblick bis zum Jahr 2025) vornehmen zu lassen ..." (S. 82)

Hintergrund dieser Empfehlung ist die zwischenzeitlich eingetretene Krise des globalen Finanzsystems mit den wirtschaftlichen Verwerfungen, die sie ausgelöst hat. Das Luftamt Südbayern ist dieser Empfehlung auch gefolgt. Es hat eine Studie zur Überprüfung der "wirtschaftlichen Grundlagen" der Intraplan-Prognose in Auftrag gegeben, wie empfohlen beim Hamburgischen WeltWirtschaftsInstitut (HWWI).

Wenn wir einen Blick auf das Verfahren unter einer klima- und damit auch energiepolitischen Perspektive werfen, dann fällt in der Empfehlung der Gutachter von der TUHH auf, dass, neben der wirtschaftlichen Entwicklung und damit der Kaufkraftentwicklung, im besonderen und lediglich auf die Ölpreise abgestellt wird. Der Sinn ist offenkundig: Die Ölpreise sind insofern ein luftfahrtspezifischer Indikator, als sie für die die Flugbetriebskosten in $\mathrm{Zu}$ kunft beeinflussenden Treibstoffpreise einen wesentlichen Faktor darstellen. Auffällig ist aber schon dessen Hervorhebung, denn es gibt etliche Faktoren, die die Flugkosten beeinflussen für die Hervorhebung muss es einen Anlass geben. Der wird von den Autoren der TUHH nicht genannt. Man wird aber unterstellen dürfen, dass der Anlass in Erwartung einer wesentlichen Änderung dieses Faktors liegt, die ihn unter sämtlichen Kostenfaktoren hervorhebt. 
Daher darf gefolgert werden, dass die öffentliche Diskussion um das Ende günstig zu fördernden Öls (,peak oil'), also eine Knappheitsdiskussion, der Anlass für die Hervorhebung ist. Formuliert man das Gemeinte so pedantisch explizit, fällt deutlich ein Mangel auf: Die äquivalente im Raum stehende Knappheit, jene, die mit dem Klimaproblem signalisiert wird, ist von den Qualitätssicherern der TUHH nicht thematisiert worden.

Man darf wohl unterstellen, dass diese Konzentration auf lediglich einen luftfahrtspezifischen Parameter und damit die Ausblendung der Implikationen des Klimaproblems dem HWWI von Seiten des Auftraggebers vorgegeben wurde - im Einklang mit dem Wortlaut des Gutachtens der TUHH. Der Wortlaut des Auftragsschreibens wurde im HWWI-Gutachten nicht mit abgedruckt, dieses Qualitätsmangels wegen ist der Leser genötigt, zu Unterstellungen zu greifen (Notabene: Der Nicht-Abdruck steht im auffälligen Gegensatz zu den Usancen in den USA, wo es üblich ist, dem ,Antwort'-Charakter einer wissenschaftlichen Expertise entsprechend, das Auftragsschreiben mitzupublizieren. Und nur das ist sachgemäß).

\section{Umgang mit der Klimapolitik als Teil der wirtschaftlichen Randbedingungen des zu- künftigen Luftverkehrs im HWWI-Gutachten - Ambivalenzen}

In der im Januar 2010 abgeschlossenen und im April 2010 der Öffentlichkeit vorgelegten HWWI-Studie ${ }^{3}$ kommt als relevant für die "wirtschaftlichen Grundlagen" des erwartbaren Luftverkehrsaufkommen lediglich der Preis des im Luftverkehr üblichen Treibstoffs Kerosin vor. Die Anlage des Gutachtens legt also nahe, dass für die Flugbetriebskosten allein die Treibstoffpreise ein relevanter Parameter sind. Als Indikator dafür werden die Rohölpreise genommen.

Nun sieht sich die menschliche Gesellschaft aber bekanntlich einer Klimaherausforderung gegenüber und es besteht im globalen Konsens die Absicht, dieses Problem zu lösen. Bedingung einer Lösung des Problems ist, die Nutzung von Kraftstoffen aus fossilen Quellen mit so hohen Kosten zu belegen, dass die Alternativen in hinreichendem Ausmaß mit hinreichender Schnelligkeit zum Durchbruch kommen. Für diesen Politikansatz steht ,der Emissionshandel'. Unter seinem Regime führen CO2-Emissionen zu steigenden Kosten proportional zum CGehalt im Kraftstoff - sofern nur der Flugverkehr betrachtet wird also proportional zu den Kerosinmengen. Der Emissionshandel ist nur ein Instrument. In Wirklichkeit wird natürlich nicht alles auf die Karte eines einzigen politischen Instruments gesetzt. Aber was auch immer politisch getan wird: Es führt zu einer Erhöhung der Kosten des Flugverkehrs.

Zu erwarten ist somit: In Zukunft werden die treibstoffbedingten Flugbetriebskosten sich anders als heute konstituieren. Sie werden sich in Zukunft aus mindestens zwei Elementen zusammensetzen, aus (a) den ölpreisabhängigen Treibstoffkosten und (b) den CO2-RechteKosten als Ausdruck der Knappheit der Erdatmosphäre. Beide Elemente beeinflussen die Flugbetriebskosten in analoger Weise, der Unterschied liegt lediglich im Subjekt der Macht: (a) liegt in den Händen der Ölförderländer, (b) in den Händen der EU. Wollte man ganz genau sein, dann müsste man in Europa noch den zu erwartenden Einfluss aus den Anforderungen einer novellierten Treibstoff-Qualitäts-Richtlinie der EU hinzuaddieren - damit wird den

\footnotetext{
3 Die Links zu den Gutachten:

http://www.regierung.oberbayern.bayern.de/aufgaben/wirtschaft/luftamt/planfeststellung/
} 
Treibstoffherstellern und Händlern vorgeschrieben, die ,Klimaqualität' von Treibstoffen zu verbessern und höhere Qualität führt bekanntlich zu höheren Kosten.

Blickt man nun genau in das HWWI-Gutachten, in dem die zukünftige klimapolitische Adressierung des Flugverkehrs ausgeblendet zu bleiben hatte, in dem somit auftragsgemäß die Klimapolitik (der EU) nicht vorkommen sollte, sondern nur die Politik der Ölförderländer, so scheint den Autoren diese ihnen aufgetragene Asymmetrie doch etwas ,gewagt' vorgekommen zu sein.

Das offenbart jene Stelle im Text, an der die Ausblendung der klimapolitischen Adressierung des Flugverkehrs explizit angesprochen wird. Die vollzogene Einbeziehung des internationalen Luftverkehrs in das klimapolitische Zentralinstrument der EU, den Emissionshandel, wird von den Autoren jedenfalls korrekt gesehen; auch wird korrekt gesehen, dass dies die Flugbetriebskosten (wie Energiekosten) sämtlicher Flüge erhöhen werde. Die Stelle lautet:

„Die Einführung von CO2-Emissionszertifikaten für Flüge von und nach Flughäfen der EU ab 2012 hat, anders als etwa bei einer Mineralölsteuer, keine direkten Auswirkungen auf die Treibstoffpreise, erhöht aber die Flugkosten.“

Das ist ein, wenn auch zarter, Hinweis an die Leitung des Qualitätssicherungsprozesses, dass die Fragestellung im Gutachten ausblendend formuliert ist. Gleich anschließend aber wird die erwähnte Erhöhung der Flugkosten aufgrund der faktischen Einbeziehung in den EUEmissionshandel gewissermaßen, weggedrückt'. Das geschieht mit Hilfe eines doppelten ,Arguments':

„Die Kosten für den Kauf der handelbaren Zertifikate halten sich zunächst in Grenzen: im Jahr 2012 werden, auf Basis der Emissionen im Jahr 2010, 85 \% der Emissionsberechtigungen kostenlos und $15 \%$ im Wege einer Versteigerung ausgegeben." (S. 8)

\section{Und:}

„Hinsichtlich der Energie- und Umwelt-/Klimapolitiken der Verbraucherländer wird in den drei Szenarien „business as usual“" unterstellt, d.h. im Prognosezeitraum gibt es keine deutlich über die bisher beabsichtigten Maßnahmen hinausgehenden Anstrengungen ..." (ebd.)

Argument (1) sagt: Die Erhöhung ist zwar eine Erhöhung, ist aber so klein, dass sie nahe Null ist. Das ist, ohne quantitativ zu argumentieren, ein ,riskanter' Schluss - er kann sich leicht als unberechtigt herausstellen. Man kann ja rechnen. Tut man das, so ist das Ergebnis:

Die Gutachter des HWWi erwarten einen ölpreisbedingten Anstieg der Kosten der Einsatzes von Treibstoff in Höhe von 9 bzw. 13 Euro pro Energieeinheit (Gigajoule, EUR/GJ). Der umgerechnete Wert einer, im Gutachten weggedrückten, CO2-Abgabe in Höhe von 30 Euro pro Tonne (EUR/t) CO2 führt zu Treibstoffverbrauchs-Mehrkosten von gut 2 EUR/GJ, ein Wert von $100 \mathrm{EUR} / \mathrm{t}$ CO2 auf 7,5 EUR/GJ. Relativ zu 9 bzw. 13 EUR/GJ sind 2 bzw. 7,5 EUR/GJ - also eine Größenordnung, die offenkundig nicht nahe Null ist und also nicht einfach vernachlässigt werden darf.

4 Das Berichtete ist in einigen Punkten nicht ganz korrekt, unterschätzt in der Tendenz die durch die luftfahrtpolitische Regelung geschaffene Knappheit an $\mathrm{CO}_{2}$-Rechten. (1) Basis der zugeteilten Emissionen ist die Phase 2004 bis 2006; (2) davon werden $95 \%$ bereitgestellt - und davon werden (3) $15 \%$ kostenpflichtig zugeteilt. Stellt man das Wachstum des EU-Luftverkehrs von 2005 bis 2010 in Rechnung, so ist die Knappheit aufgrund dieser Regelung, korrekt rezipiert, doch deutlich höher als auf Basis der Darstellung des HWWI zu vermuten (vgl. Luhmann 2008). 
Argument (2) ist nichts anderes als ,weiße Salbe': Wenn schon die realisierten Maßnahmen faktisch unberücksichtigt bleiben, wie soll dann die Aussage gefüllt werden, die besagt, dass darüber hinaus „,beabsichtigte Maßnahmen“ berücksichtigt werden? Zudem ist da der merkwürdige exkulpierende Hinweis auf den „Prognosezeitraum“ bis 2020 bzw. 2025, der zwar auftragsgemäß aber der beabsichtigten Nutzungsdauer des Anlasses der Untersuchung grotesk unangemessen ist. Die ,Umsetzung' von Argument (2) im Gutachten ist im Einklang mit dem Auftragsschreiben seitens der Genehmigungsbehörde entschieden worden: Klimapolitik mit Auswirkung auf den Flugverkehr vom Boden des Freistaates Bayern aus findet nicht statt. Bayern ist gleichsam als Faradayscher Käfig am unteren Rand des Mehrebenensystems Land - Bund - EU - UN: ,Oben' oder jenseits von 2020 mag zwar fleißig Klimapolitik betrieben werden, Bayern bzw. der Flughafen München jedoch werden als eine Enklave vorgestellt, die davon ,,im Prognosezeitraum“ unberührt bleibt. Im Umkehrschluss und etwas zugespitzt formuliert bedeutet das:

Die dritte Startbahn in München ist nur unter der Voraussetzung notwendig, dass das Klimaproblem praktisch negiert wird.

Das ist die Botschaft des wissenschaftlichen Gutachtens zur Begründung der wirtschaftlichen Rahmenbedingungen, unter denen der erwogene Bau einer dritten Startbahn beim Flughafen München gerechtfertigt ist - wenn es auch vermutlich bei freier Entscheidung der Gutachter nicht so angelegt worden wäre.

Es wäre zu erwarten gewesen, dass die Gutachter mit der Genehmigungsbehörde Rücksprache halten in Bezug auf die Reichweite ihres Auftrags und die Frage aufwerfen, ob die Ausblendung jeglicher Klimapolitik gerechtfertigt sei. Das haben sie offenbar versäumt. So kommt der methodisch interessierte Leser bei der Lektüre des Gutachtens nicht auf seine Kosten, wenn er aus Neugier in einem konkreten Fall nachschauen möchte, wie mit dem ,Risiko', das die Klimapolitik für die Wachstumsträume der Luftverkehrsbranche und also für den Auftraggeber der Intraplan-Prognose darstellt, in einem solchen Gutachten professionell umgegangen wird. Es stellt ja trotz Klimapolitik eine wachsende Nachfrage nach Flugverkehrsleistungen fest. Wieso die Resultante der widerstrebenden Kräfte bei Wirtschaftswissenschaftlern ein positives, ein Wachstums-Vorzeichen, bekommt, das scheint des Studiums wert.

Das Ergebnis der Durchsicht ist jedoch, wie angedeutet, eher trivial: Das positive Vorzeichen wird dadurch konstruiert, dass Klimapolitik in dem Gutachten nicht stattfindet. Von den in Wirklichkeit widerstreitenden Tendenzen ist eine ausgeblendet worden.

\section{Nachbesserungen in ,last minute'-Manier}

Dieser ausblendende Zugriff ausgerechnet im Rahmen des ,Qualitätssicherungsprozesses' kann nur als desaströs und im Hinblick auf den Zweck im Rahmen eines Planfeststellungsverfahrens widersinnig bezeichnet werden. Das scheint den Verantwortlichen dann doch noch im letzten Moment deutlich geworden zu sein. Wäre das Ergebnis nämlich tatsächlich unter dem Titel ,Die dritte Startbahn in München ist nur unter der Voraussetzung notwendig, dass das Klimaproblem negiert wird' in die Schlagzeilen geraten, so wäre der Qualitätsmangel erwiesen und der Gutachtensprozess hätte von vorne neu beginnen können: Für die am Bau Interessierten wären etliche Jahre ,in den Sand gesetzt" worden.

Um diese Blamage zu verhindern haben die Akteure die Ausarbeitung „Ergänzende Szenariobetrachtungen zur Luftverkehrsprognose 2020 für den Flughafen München“"gewählt, die der 
kommerzielle Prognoseersteller, Intraplan Consult GmbH, in Reaktion auf das HWWIGutachten noch anzufertigen hatte. ${ }^{5}$

Das Mandat und die Aufgabenstellung für diese abschließende Ausarbeitung in Reaktion auf den Qualitätssicherungsprozess wird, wieder lediglich vom Auftragnehmer, wie folgt referiert: Das Luftamt Südbayern habe der Flughafen München GmbH mit Schreiben vom 23. 9. 2009 signalisiert, dass die Ergebnisse des „Wirtschaftsgutachtens“ (also der HWWIUntersuchung), in einem zweiten Schritt in das Rechenmodell der Luftverkehrsprognose zu integrieren seien." Nachdem das HWWI-Gutachten am 26. 1. 2010 übergeben worden war, hat Intraplan diesen Auftrag mit Vorlage der überarbeiteten Prognose erfüllt. Nimmt man den Auftrag wörtlich, besagt er, die Ausblendung der Klimapolitik als wirtschaftliche Randbedingung sei beizubehalten.

Intraplan hat aber nicht nur dem expliziten Auftrag entsprochen. In den ,Ergänzenden Szenariobetrachtungen' finden sich vielmehr auch eine explizite und durchaus kompetente Auseinandersetzung mit den prospektiven Kostenfolgen der bereits erfolgten Einbeziehung des Luftverkehrs in den Europäischen Emissionshandel. Damit geht diese abschließende Prognose deutlich über ihren expliziten Auftrag hinaus. Sie füllt somit zumindest einen wesentlichen Teil dessen auf, was an Lücken bezüglich des Klimaproblems den Begutachtungsprozess bis dahin kennzeichnete - in der Verantwortung der Leitung des Qualitätssicherungsprozesses, des Luftamtes Südbayern.

Die entscheidende Qualitätsverbesserung der Nachfrageprognose ist somit, etwas paradox aber nicht ungewöhnlich, außerhalb dieses Prozesses herbeigeführt worden - vermutlich auf Veranlassung des Auftraggebers der ,Ergänzenden Szenariobetrachtungen', der Flughafen München $\mathrm{GmbH}$. Irgendwer muss eben drauf kommen und irgendwer hat den zusätzlichen Aufwand zur Schließung dieser klaffenden Lücke zu bezahlen.

Den Verantwortlichen des Flughafens München, so kann man schließen, war die Bedeutung der Klimathematik im Vorfeld der ersten Auftragsvergabe, also vor 2007 und damit vor dem ,Hype' des Themas nach Vorlage der drei IPCC-Gutachten im Laufe des Jahres 2007, noch nicht als relevant vor Augen - im Jahr 2010 war das anders.

\section{Ergebnis zum Flughafen München}

Inhaltlich errechnet Intraplan für die Einbeziehung des Luftverkehrs in den Emissionshandel (im Basisszenario) Mehrkosten von rund 100 Euro pro Tonne Kerosin bzw. 18\% der Treibstoffkosten - auf Basis eines CO2-Preises von 30 Euro pro Tonne. Das entspricht 3,2 \% der Gesamtkosten (bis 2025) und, da diese Kostenkategorie neu ist, einem Kostenveränderungsanteil in etwa gleicher Höhe. Dieser Wert von 3,1\%-Punkten wird in der überarbeiteten Prognose ins Verhältnis gesetzt zu der Veränderung von weiteren Flugkostenkomponenten.

Da ist einerseits die Veränderung aus dem Anstieg der Rohölpreise, mit 17,1 \%-Punkten (bis 2025), da ist andererseits die (negative) Veränderung aus einer technisch herbeizuführenden Erhöhung der Treibstoffeffizienz in Höhe von minus 9,7 \%-Punkten. ${ }^{6}$ Relativ dazu, das ist damit von den Intraplan-Autoren deutlich gemacht, erscheint die Bedeutung des Effekts aus

\footnotetext{
5 Erstellungsdatum: 10. März 2010; Publikationsdatum: unbekannt, vermutlich Mai 2010. Verfügbar unter https://www.muc-ausbau.de/downloads/aenderungen_PFV/01_LVP-PrognoseErg_nzung.pdf

6 Alles in Tab. 2-4
} 
dem neuen Klimaschutzinstrument klein. Und mehr an Klimapolitik nimmt die abschließende Prognose eben nicht in den Blick.

Offenkundig gilt somit: Die Einbeziehung des Luftverkehrs in den Europäischen Emissionshandel bringt zwar einen Kostenbeitrag, aber offenbar keinen entscheidenden. Er wird, so die Erwartung der Flughafenbetreiber, schon durch die Hebung der Energieeffizienzreserven, die die Flugzeughersteller nun ausschöpfen werden, mehr als ausgeglichen werden und somit in der Wirkung auf die Gesamtkosten, verdampfen'. Auf die Wachstumsdynamik des Flugverkehrs in München wird dieses Instrument keinen Einfluss in einer solchen Größenordnung haben, dass das Vorzeichen der bisherigen Entwicklung gekippt wird. Das zu erwarten, so zeigt die Einbindung des Kosteneffekts aus der Einbeziehung des Luftverkehrs in den Emissionshandel in eine Betrachtung sämtlicher Kosten in dieser Prognose, wäre illusorisch. Das ist die Leistung der Intraplan-Ausarbeitung: Im Grunde zeigen die Relationen, die die Intraplan-Prognose ausweist, wie bescheiden der Anspruch der Verbraucherländer mit ihrem Klimaschutzbekenntnis doch eigentlich ist, wenn man ihn mit dem vom Flughafenbetreiber ,akzeptierten' zusätzlichen Abschöpfungsanspruch auf Erdölrenten seitens der Erdölproduzenten in Höhe eines Faktors von mehr als dem Fünffachen ${ }^{7}(!)$ vergleicht.

Die Feststellung, dass Intraplan die prospektiven Kostenfolgen der bereits erfolgten Einbeziehung des Luftverkehrs in den Europäischen Emissionshandel kompetent analysiert habe, bedeutet aber natürlich nicht, dass damit die politische Reaktion auf die Großherausforderung ,Klimawandel' in angemessener Weise prospektiv berücksichtigt worden sei. Weitere Ausblendungen finden sich, von denen lediglich ein paar Beispiele genannt seien:

Die im politischen Raum vorhandene Überzeugung, dass nur bei einem Preis von (mindestens) 70 EUR/t CO2 die von der EU beschlossenen Minderungsziele erreichbar sind.

Der mögliche interregionale Effekt, also auf das Flug-Aufkommen in München, dadurch, dass Dubai die Funktion eines Luftfahrtkreuzes (Hub) aufzubauen im Begriffe ist, dessen Position durch die EU-Klimapolitik im Flugbereich gefördert werden könnte.

Die Flugsteuerpläne der Bundesregierung, die für Passagiere Kosten bringen, die deutlich über die aus der Einbeziehung in den EU-Emissionshandel hinausgehen und zudem, der Struktur wegen, insbesondere im innerdeutschen Verkehr dämpfend wirken könnten - daraus ist ein Effekt auf die Kapazitätsnachfrage zu erwarten, der weit überproportional ist im Vergleich zu dem aus der autonomen Nachfrage von Passagieren von und nach München.

Das sind Ausblendungen, die hier auf einer sehr niedrigen Konkretionsstufe formuliert sind. Hilfreich könnte auch sein, einen Standort weiter oben auf der Beobachtungsleiter einzunehmen und einen Blick auf die systemischen Prozesse rund um den Ausbau von Flughäfen zu werfen, worum die soziologische Verkehrswissenschaft sich bemüht (z.B. Kesselring 2009).

\section{Fazit}

Die Kraft des Anstiegs der Flut ist enorm und wird mit den Mitteln des Emissionshandels (zumindest nach bisherigen Konzepten) nicht einzudämmen sein. Der Imperativ einer massiven Reduktion des Treibstoffausstoßes aber ist nicht verhandelbar und kann nicht aufgegeben werden. Es bleibt nur, ein Konzept zu etablieren, das Verkehr und Verkehrsinfrastruktur nicht

$7 \quad 17,1 / 3,1=5,5$ 
mehr getrennt betrachtet, und also das Verkehrswachstum nicht als naturgegebene Notwendigkeit begreift.

Aussicht auf Erfolg hat das nur, wenn die Gründe für die Flut korrekt diagnostiziert wurden. Ein Kernelement für die steigende Verkehrsflut liegt im Genehmigungsverfahren für Verkehrsinfrastrukturen. Eine Abschaffung der bisherigen Privilegierung von Verkehrswachstum als eigenständiger und extern induzierter Größe im Rahmen von Planung und Genehmigung von Verkehrsinfrastrukturprojekten bietet sich somit als Gegenmittel an.

Dieses Plädoyer wird auf Skepsis stoßen. Mit ihm ist unter den vielen Ursachen, welche dem Phänomen ,Verkehrsflut‘ zu Recht zugeschrieben werden können, nämlich eine einzige herausgegriffen worden. Der Ansatz im Genehmigungsverfahren für Verkehrsinfrastrukturen erscheint wie der sprichwörtliche Stein, mit dem David den Goliath besiegen wollte. Bei einem Phänomen, das viele Ursachen hat, erscheint das zudem als ein Akt willkürlicher Auswahl. Denkt man Kausalität linear, erscheint das als ein wenig aussichtsreicher Ansatz.

Kausalität ist aber nicht linear zu denken. Jedenfalls ist dies das Heisenbergsche Verständnis von ,Revolution“: Revolutionen sind nicht-linear. Heisenberg hat gelegentlich das Kriterium formuliert, das ihn bei seiner Forschung geleitet hat: Bei der Auswahl eines unter vielen Parametern, welche ein System beeinflussen, sei es ihm um den kleinstmöglichen gegangen, mit dem eine Veränderung des ganzen Systems ( = ,Revolution') zu erreichen sei. Ob man dann, folgt man diesem Kriterium, richtig getippt hat bei der konkreten Auswahl, kann nur die Erfahrung des Ausprobierens erweisen.

\section{Literatur}

Canzler, Weert, Andreas Knie, 1998: Möglichkeitsräume. Grundrisse einer modernen Mobilitäts- und Verkehrspolitik, Böhlau Vlg., Wien/Köln/Weimar

Freudendahl-Pedersen, Malene, 2005: Structural Stories, Mobility and (Un)freedom, in:

Thomsen, Thyra Uth, Lise Drews Nielsen and Henrik Gudmundsson (Eds.): Social Perspectives on Mobility, Farnham/UK Ashgate, p. 29-45

Hartwig, Heine/Mautz, Rüdiger/Rosenbaum, Wolf, 2001: Mobilität im Alltag. Warum wir nicht vom Auto lassen. Campus Vlg.,New York/Frankfurt/M.

Kesselring, Sven 2009: Global Transfer Points: The Making of Airports in the Mobile Risk Society. In: Cwerner, Saulo; Kesselring, Sven; Urry, John (Hg.): Aeromobilities. London, New York N.Y.: Routledge (International library of sociology), S. 39-60.

Kuhm, Klaus, 1997: Moderne und Asphalt. Die Automobilisierung als Prozess technologischer Integration und sozialer Vernetzung. Centaurus Vlg., Pfaffenweiler Luhmann, Hans-Jochen, 2008: Das statuierte Exempel: Die EU reguliert internationale Luftfahrt hinsichtlich ihres Klimaeffekts unilateral. In: Zeitschrift für Energiewirtschaft (ZfE), Jg. 32; H. 4, S. $230-234$.

Rammler, Stephan, 2001: Mobilität in der Moderne. Geschichte und Theorie der Verkehrssoziologie, edition Sigma, Berlin

Rat von Sachverständigen für Umweltfragen (SRU), 2005: Umwelt und Straßenverkehr. Hohe Mobilität - Umweltverträglicher Verkehr. Sondergutachten, Wiesbaden

Urry, John, 2007: Mobilities. Polity Press, Cambridge Verron, Hedwig et al. 2006: Determinanten der Verkehrsentstehung. (UBA-Texte 26/05) Berlin 
Winter, Gerd, 2010: Lob des Flaschenhalses. Über Verkehrsbegrenzung durch Straßenplanungsrecht. In: Dolde et al. (Hg.): Verfassung - Umwelt - Wirtschaft: Festschrift für Dieter Sellner zum 75. Geburtstag. München, Beck Vlg. S. 193 - 206 